\title{
Implantação de comissão de ética de enfermagem institucional: buscando a prática com equidade
}

\section{Implementing a nursing ethical practice commission in an university hospital: searching for practice with equity}

DOI: $10.46814 /$ lajdv3n3-018

Recebimento dos originais: 01/05/2021

Aceitação para publicação: 30/06/2021

\author{
Elizabeth Pimentel Da Silva \\ Enfermeira/Mestre Em Enfermagem Em Bioética, Ética Aplicada E Saúde Coletiva Pelo \\ Ppgbios/Ufrj \\ E-mail: elizabethpimente1030372@gmail.com \\ Rafael Barroso Gaspar \\ Enfermeiro/Mestre Em Enfermagem Pela Eean/Ufrj \\ E-mail: rafaelb.gaspar@gmail.com \\ Fábio Gonçalves Ferreira \\ Enfermeiro/Mestrando Em Bioética, Ética Aplicada E Saúde Coletiva Pelo Ppgbios/Uerj \\ E-mail: enfermeirodomar@hotmail.com \\ Valda Maria De Almeida \\ Enfermeira E Bacharel Em Direito \\ E-mail: valdasoares43@gmail.com \\ Juliana De Souza Silva \\ Enfermeira/mestranda em saúde coletiva pela UFRJ \\ E-mail: julianaernando@gmail.com \\ Patrícia De Souza Veiga \\ Enfermeira/Especialização Em Estomaterapia Pela Uerj \\ E-mail: patriciadesouza.john@gmail.com \\ Ivaldo Dos Santos Pereira
ENFERMEIRO
dinhofederal2@gmail.com \\ Claudia Paiva De Oliveira \\ Técnica De Enfermagem/Graduanda Em Gestão Hospitalar \\ E-mail: claudiamaudia@gmail.com
}

\section{RESUMO}

Objetivo: Descrever a experiência da implantação da Comissão de Ética de Enfermagem do Hospital Universitário Clementino Fraga Filho. Métodos: Trata-se de estudo descritivo, tipo relato de experiência, construído a partir da vivência dos autores. Para a organização do conteúdo, foram utilizadas análise documental, entrevistas com os membros da comissão e revisão dos processos 
analisados. Os resultados foram divididos em histórico da formação, estruturação e organização, além de atividades realizadas. Resultados: A comissão teve início em 2017, tendo como membros atualmente nove profissionais de enfermagem. Quanto à estruturação e organização, coube aprovação de regimento interno, confecção de material de divulgação, implantação da caixa de denúncias e visita aos setores. Entre as atividades realizadas, destacam-se a participação em eventos sobre ética e legislação; acolhimento e orientação de profissionais; sindicância e conciliação de casos; elaboração de pareceres; assessoria para a Divisão de Enfermagem; elaboração de relatórios e capacitação dos membros. Conclusão: É importante dar voz aos trabalhadores da enfermagem para que seja construída uma prática ética, tratando com respeito os problemas morais frequentemente vivenciados. A implantação da comissão foi um passo em busca da equidade de tratamento entre os profissionais ouvindo, registrando e direcionando soluções com base na ética profissional elevada.

Palavras-chave: Comissão de Ética, Ética em Enfermagem, Legislação de Enfermagem.

\begin{abstract}
Objective: Describe the experience of implementing a Nursing Ethical Practice Commission in the University Hospital Clementino Fraga Filho. Methods: This is a descriptive study, such as an experience report, constructed based on the experience of its authors. To organize the content of the study, document analysis, interviews with the members of the commission and review of the analysed complaints were utilised. The results were divided by history of the formation, structure and organization, besides the activities performed by the commission. Results: The commission was founded in 2017, currently, having nine nursing professionals as members. As in regards to structure and organisation, it approved internal regulations, produced material for divulgation, implemented a box of complaints and visited the sectors. From the realised activities, the highlights are: the participation in events about ethics and legislation; reception and orientation to professionals; case mediation and internal affairs investigation; legal opinion development; advisory procedures for the Nursing Department; report development and member's training. Conclusion: It is important to give voice to nursing staff in order to build an ethical practice, treating with respect the moral problems frequently experienced. The implementation of the commission was a step towards equity in treatment between staff by listening, registering and proposing solutions based on a highly elevated professional ethic.
\end{abstract}

Keywords: Ethics Committees; Ethics, Nursing e Legislation, Nursing.

\title{
1 INTRODUÇÃO
}

A Resolução nº 593/2018, do Conselho Federal de Enfermagem (COFEN) normatizou a criação e funcionamento das Comissões de Ética de Enfermagem (CEE) nas instituições com Serviço de Enfermagem, além de prescrever que estas exercem a representação dos Conselhos Regionais de Enfermagem no âmbito das instituições onde foram instituídas. Atuando com funções educativa, consultiva, e de conciliação, orientação e vigilância ao exercício ético e disciplinar dos profissionais de enfermagem, as CEE devem estabelecer relações de autonomia e imparcialidade com os estabelecimentos de saúde, bem como resguardar o sigilo e discrição nos assuntos vinculados às condutas de caráter ético e disciplinar dos profissionais de enfermagem (CONSELHO FEDERAL DE ENFERMAGEM, 2018). 
Entre as atribuições específicas dos membros da CEE, destacam-se: representar o Conselho Regional de Enfermagem (COREN) na instituição de saúde em se tratando de temas relacionados à divulgação do Código de Ética dos Profissionais de Enfermagem; divulgar e zelar pelo cumprimento da legislação de enfermagem vigente; e identificar as ocorrências éticas e disciplinares na instituição de saúde onde a CEE atua, recebendo denúncia de profissionais de enfermagem e usuários dos serviços de saúde. Outra importante função é elaborar relatórios sobre as atividades referentes ao campo ético do exercício da enfermagem, estando estes restritos à narrativa dos fatos que ensejaram a denúncia, com a devida anexação de documentos cabíveis, desde que relativos a qualquer indício de infração ética (CONSELHO REGIONAL DE ENFERMAGEM DO RIO DE JANEIRO, 2015).

Compete também à CEE encaminhar seus relatórios ao COREN e ao Enfermeiro Responsável Técnico (RT) da instituição de saúde para o devido conhecimento dos casos em que haja indícios de infração ética ou disciplinar. Busca-se propor a participação conjunta do Enfermeiro RT e do Enfermeiro responsável pelo Serviço de Educação Permanente de Enfermagem para o desenvolvimento de ações preventivas e educativas sobre questões éticas e disciplinares, inclusive quanto à promoção de temas pertinentes à ética no âmbito multiprofissional (CONSELHO REGIONAL DE ENFERMAGEM DO RIO DE JANEIRO, 2015).

A CEE assessora a Diretoria/Chefia/Coordenadora de Enfermagem da instituição de saúde a respeito das questões éticas ligadas à enfermagem, promove a divulgação sobre as atribuições da CEE, participa das atividades educativas do COREN a que está vinculada, além de atender às solicitações de reuniões e convocações inerentes às suas atribuições. Por fim e não menos importante, compete à CEE promover e participar de treinamento e capacitação e apresentar anualmente relatório sobre suas atividades junto ao COREN (CONSELHO REGIONAL DE ENFERMAGEM DO RIO DE JANEIRO, 2015).

No que tange à Resolução no 593/2018, consta em seu artigo $11^{\circ}$ que os Conselhos Regionais de Enfermagem poderão baixar decisão aprimorando o regulamento desta norma no âmbito de sua jurisdição, principalmente o papel da comissão eleitoral e modelo de regimento da CEE, observando o disposto presente na respectiva resolução (CONSELHO FEDERAL DE ENFERMAGEM, 2018).

Nesse sentido, o COREN do Rio de Janeiro (COREN/RJ) publicou a Decisão no 1821/2012, que fixou normas gerais de instalação, eleição e procedimento de trabalho da Comissão de Ética de Enfermagem nas instituições de saúde públicas e privadas que tenham profissionais de enfermagem em seu quadro de pessoal. Cabe salientar que a implantação dos termos da referida decisão do COREN/RJ se fundamenta nos princípios básicos da democracia, da liberdade e equidade numa perspectiva de atuação conciliadora e educativa (CONSELHO REGIONAL DE ENFERMAGEM DO RIO DE JANEIRO, 2012). 
O presente estudo tem como objetivo descrever a experiência da implantação da primeira CEE do Hospital Universitário Clementino Fraga Filho (HUCFF).

\section{METODOLOGIA}

\subsection{TIPO DE ESTUDO}

Trata-se de um estudo descritivo, do tipo relato de experiência, construído a partir da vivência dos autores e da análise de documentos arquivados pela comissão.

\subsection{SUJEITOS ENVOLVIDOS NA EXPERIÊNCIA}

As reflexões dizem respeito às experiências dos membros da primeira CEE do HUCFF, através das vivências e descrição e análise das atividades desenvolvidas.

\subsection{PERÍODO DE REALIZAÇÃO DA EXPERIÊNCIA}

A descrição abarca o período de dezembro de 2017, quando foi estabelecida a primeira gestão da CEE, até a previsão de término, em julho de 2021. Ressalta-se que, em virtude das condições pandêmicas por COVID-19, houve atraso para novo processo eleitoral, estendendo esta gestão, com previsão de finalização para julho de 2021, quando a próxima comissão tomará posse.

\subsection{CENÁRIO DE ESTUDO}

Realizado no HUCFF, unidade de saúde que pertence ao complexo hospitalar da Universidade Federal do Rio de Janeiro (UFRJ) e conta atualmente com aproximadamente 1.453 funcionários lotados na Divisão de Enfermagem, sendo 376 enfermeiros, 727 técnicos de enfermagem, 315 auxiliares de enfermagem e 35 atendentes, tanto estatutários, quanto contratados.

\subsection{COLETA DE DADOS E PROCEDIMENTOS DE ANÁLISE}

Os dados utilizados constaram de análise documental, entrevistas com os membros do comitê e relatórios dos processos encaminhados e analisados pela comissão. A descrição da experiência foi dividida em três grupos: históricos da formação; estruturação e organização das atividades; e atividades realizadas. Em históricos da formação, foram resgatadas informações desde o período eleitoral até a informação da composição atual do grupo. Quanto à estruturação e organização, foram descritas as medidas implementadas para o devido funcionamento da CEE. Já para a apresentação das atividades implementadas, foi construído um quadro que mostra, de forma detalhada, por ano, as ações que fizeram parte do trabalho da comissão. 


\subsection{PROCEDIMENTOS ÉTICOS}

Acerca dos aspectos éticos, cabe ressaltar o sigilo e a descrição no relato da experiência.

\section{DESCRIÇÃO DA EXPERIÊNCIA}

\subsection{HISTÓRICO DE FORMAÇÃO}

Após visitas do COREN à unidade para sensibilização da categoria quanto à necessidade da normatização da criação da CEE do HUCFF, foi instituída uma comissão eleitoral. O pleito sucedeu a divulgação para candidatura dos profissionais interessados e encontros para discussão da finalidade e importância da criação da CEE. A divulgação do resultado, homologação e publicação em Diário Oficial ocorreram em dezembro de 2017.

Importante frisar que, feita a homologação, em virtude de modificações na Direção da Divisão de Enfermagem, houve a saída de três enfermeiros que passaram a desempenhar funções de gerência, o que poderia gerar conflito de interesses. Ao longo da gestão da CEE, foi solicitado desligamento por um técnico de enfermagem que se aposentou em 2019, e por um enfermeiro, que deixou a equipe em 2020 objetivando participação em disputa eleitoral do COREN-RJ. Sendo assim, de um total inicial pós-eleição de sete enfermeiros e seis técnicos/auxiliares de enfermagem, integram a equipe, em abril de 2021, quatro enfermeiros e cinco técnicos/auxiliares de enfermagem.

Após a posse da gestão, foram determinadas as funções dos membros, sendo que os cargos de presidente e vice-presidente estavam previamente definidos pela contagem dos votos no pleito, portanto foram eleitos pela comissão os membros para primeiro secretário, segundo secretário e vogal.

Vale informar que, desde o início, até por questões da diminuição da equipe, foi acordado que o trabalho seria desenvolvido de forma horizontal, democrática e com ampla participação de todos, titulares e suplentes, enfermeiros e técnicos/auxiliares de enfermagem, com divisão de poderes e responsabilidades.

\subsection{ESTRUTURAÇÃO E ORGANIZAÇÃO DAS ATIVIDADES}

Inicialmente, procedemos à apreciação e discussão do nosso Código de Ética -importante lembrar que, quando iniciamos o processo, estava em vigor a Resolução COFEN nº 311/2007, e em 05 de abril de 2018 passou a vigorar a Resolução COFEN nº 564/2017 (CONSELHO FEDERAL DE ENFERMAGEM, 2007, 2017). Tal mudança gerou uma necessidade de rediscussão, revisão e divulgação do novo código.

Paralelo a isso, houve a elaboração do regimento interno e dos impressos (formulário de denúncia, abertura de comissão sindicante, modelos de ata de reunião, oitiva e termo de conciliação). 
Ademais, a equipe confeccionou material de divulgação, em que se apresentaram sucintamente os objetivos da comissão; as formas de acesso à CEE; foto, nome e setor dos membros, os quais procederam à visita e difusão deste material nos setores, esclarecendo de dúvidas e propagando o trabalho que seria desenvolvido.

Foi produzida e instalada uma caixa de denúncias no subsolo do hospital, de modo a facilitar o processo de denúncia e, a fim de agilizar a comunicação, criou-se um e-mail para a CEE com divulgação entre os pares.

Houve também a solicitação para a Divisão de Enfermagem de uma sala para a comissão, para que esta pudesse se reunir e receber os profissionais de forma confortável e particular, além de computador, impressora, mobiliário, linha telefônica e livros para ata e protocolo.

\subsection{ATIVIDADES EDUCATIVAS}

Como processo de educação continuada da equipe, realizou-se a divulgação do novo Código de Ética dos Profissionais de Enfermagem à Direção da Divisão de Enfermagem e às Chefias de Serviço, para que se tornassem elementos propagadores das informações, assim como foram feitas visitas aos setores. Em 2019, o COREN/RJ lançou um aplicativo para atualização das legislações pertinentes à categoria, o qual foi amplamente divulgado pela CEE para a equipe de enfermagem do HUCFF.

Dentre os eventos de cunho educativo para a equipe de enfermagem do hospital, destacam-se a participação do grupo como comissão organizadora, realização de palestras e dinâmicas de grupo do evento "Ética e Legislação - Reflexões e Desafios na prática de Enfermagem", junto à Coordenação de Educação Permanente, com simulação de julgamento ético para os profissionais do hospital.

Desenvolveu-se, desde o início, parceria com o Serviço de Saúde do Trabalhador do HUCCF para a discussão de demandas conjuntas e possibilidade de ajuda mútua no encaminhamento dos casos.

As atividades educativas extramuros incluíram orientações sobre a implementação e as atividades desenvolvidas pela CEE para enfermeiros atuantes em outras instituições da rede privada e pública do estado do Rio de Janeiro.

\subsection{DEMANDAS PROFISSIONAIS}

O Quadro 1 mostra as demandas profissionais enfrentadas pela comissão de acordo com os anos de trabalho - 2018, 2019, 2020 e 2021 -, referentes às seguintes atividades descritas: acolhimento, escuta ativa e orientação de funcionários; abertura de processos éticos; pareceres; conciliações; elaboração de relatórios; assessoria para a Divisão de Enfermagem/coordenadores da assistência; solicitação de desagravo e encaminhamentos para o COREN-RJ. 
Quadro 1 - Atividades desenvolvidas pela CEE de 2018 a 2021

\begin{tabular}{|l|c|c|c|c|}
\hline Atividades Desenvolvidas & $\mathbf{2 0 1 8}$ & $\mathbf{2 0 1 9}$ & $\mathbf{2 0 2 0}$ & $\mathbf{2 0 2 1}$ \\
\hline $\begin{array}{l}\text { Acolhimento, escuta ativa e orientação de } \\
\text { funcionários }\end{array}$ & 06 & 02 & 08 & 03 \\
\hline Abertura de processos éticos & 02 & 02 & 04 & 02 \\
\hline Conciliações de & 01 & & 03 & 01 \\
\hline Elaboração de relatórios para Divisão & - & 04 & 07 & 01 \\
\hline $\begin{array}{l}\text { Assessoria } \\
\text { Enfermagem/Coordenadores }\end{array}$ & 02 & - & 01 & - \\
\hline Pareceres & - & 01 & 01 & - \\
\hline Solicitação de desagravo & 01 & 03 & 01 & - \\
\hline Encaminhamento para o COREN/RJ & & & - & - \\
\hline
\end{tabular}

Fonte: CEE/HUCFF.

Estes dados indicam um número relevante de acolhimento, escuta ativa e orientação de funcionários, totalizando 19 casos, que não necessariamente se converteram em abertura de processos éticos (10 processos). Isso mostra que, depois do acolhimento, as dúvidas dos profissionais puderam ser sanadas, apontando para outros tipos de conduta pelo profissional.

É importante acrescentar que, aberto o processo ético, é instaurada uma comissão sindicante para a apuração dos fatos e realização das intimações e oitivas necessárias. Findos os trâmites, havendo possibilidade conciliatória, os envolvidos são chamados e, após conversa e acordo, assinam o termo de conciliação. As intimações, oitivas e termos são entregues assinados para os denunciantes e denunciados. Quando não há chance de conciliação pelas partes, o caso é encaminhado para que o COREN/RJ lhe dê continuidade. Independente do desfecho, é confeccionado um relatório final do acompanhamento do caso pela comissão.

Dos 10 processos instaurados, cinco resultaram em conciliação, três tiveram encaminhamento para o COREN/RJ e dois ainda estão em andamento. O Quadro 1 mostra mais encaminhamentos para o COREN/RJ em 2019 do que abertura de processos éticos, pois outras demandas podem ter esse fim, como o caso de solicitação de desagravo ou casos que ultrapassam a capacidade de resolutividade da CEE, por se tratar de situações mais graves.

Além dos processos, foi realizada assessoria para a Divisão de Enfermagem ou gerências (quatro), bem como redação de pareceres de acordo com as demandas (três). Quanto à elaboração de relatórios, são redigidos anualmente e ao final de cada tramitação instaurada pela CEE.

\subsection{CAPACITAÇÃO DA EQUIPE}

Logo após o início dos trabalhos na CEE, a equipe participou no I Encontro da Comissão de Ética de Enfermagem do Instituto Nacional de Traumatologia e Ortopedia e no curso intitulado "Ética e Legislação" do projeto Capacita COREN-RJ. 
Já dentro dos muros do HUCFF, enfrentamos desafios de aprendizado como comissão organizadora, palestrantes e atores em oficina no evento "Ética e Legislação - Reflexões e Desafios na prática de Enfermagem". Ocorreu também a participação de componente da CEE em um curso de Segurança do Paciente, em que várias questões com relação à ética foram abordadas, sendo repassadas e discutidas com os demais integrantes.

Observou-se a necessidade de busca de maior conhecimento para a realização das atividades da CEE com eficiência e segurança. Desse modo, dois componentes da comissão ingressaram no mestrado do Programa de Pós-Graduação em Bioética, Ética Aplicada e Saúde Coletiva (PPGBIOS) no intuito de auxiliar no subsídio das questões éticas a serem enfrentadas. Quanto à participação em eventos com apresentação de trabalhos produzidos pela equipe, destacam-se: participação na Semana de Enfermagem do HUCFF/UFRJ/2019 na modalidade de pôster dialogado; participação no 57º Congresso Científico do Hospital Universitário Pedro Ernesto/Universidade do Estado do Rio de Janeiro (HUPE/UERJ) e no VII Seminário de Integração dos Servidores Técnico-Administrativos em Educação (SINTAE), ambos na qualidade de expositores de pôster.

Ainda em se tratando da divulgação científica dos trabalhos da comissão, houve a confecção e submissão do trabalho "Relato de experiência da implantação da comissão de ética de enfermagem do HUCFF - em busca da prática com equidade" no $72^{\circ}$ Congresso Brasileiro de Enfermagem" e desenvolvimento e submissão de artigo para periódico científico, modalidade relato de experiência: "Implantação de comissão de ética de enfermagem institucional: buscando a prática com equidade".

\section{LIMITAÇÕES DA EXPERIÊNCIA}

Houve uma dificuldade inicial na alocação de sala para a CEE, por dificuldades institucionais de se obter um espaço adequado; logo ocorreu um atraso no arquivamento de materiais e ambiente adequado para reuniões, escuta e acolhimento.

Uma importante observação se refere ao fato de a comissão ser formada por membros atuantes em plantões, turnos e funções diferentes, o que dificulta o encontro e atendimento às demandas, lembrando que os integrantes devem cumprir a carga horária de trabalho normal, não devendo ocorrer nenhum tipo de benefício monetário ou folgas para executar o trabalho na comissão. A impossibilidade de obter auxílio de um secretário externo ou funcionário para encaminhamentos, devido ao caráter sigiloso das ações, por vezes sobrecarrega os membros com atividades administrativas.

A saída de membros da CEE por motivo de ocupação de cargos de chefia, aposentadoria ou participação em pleito eleitoral, acabou por acarretar também uma maior demanda para os componentes da comissão. 
O HUCFF, assim como todos os estabelecimentos de assistência à saúde, passa por um momento de reestruturação dos serviços face à pandemia de COVID-19; esse cenário mostrou-se um dificultador para a realização dos encontros ordinários e extraordinários. Tentou-se lidar com essa nova perspectiva criando estratégias virtuais, que, embora muito úteis, ainda não são de domínio de todos os membros da CEE.

Outra limitação atribuída à pandemia foi o afastamento temporário de alguns membros por adoecimento pessoal e de familiares, ou aumento da carga de trabalho por atuação direta na assistência aos pacientes com COVID-19. Desse modo, o grupo, que já estava em número menor do que foi eleito, se tornou, em alguns momentos, mais diminuto. Tal situação se refletiu na dificuldade para montar as comissões necessárias, visitas, oitivas e demais demandas. Vale ressaltar a sobrecarga de estresse físico e mental para os integrantes que têm atuado com as mudanças provocadas pela pandemia, podendo gerar transtornos para o ideal cenário de resolução de problemas.

\section{CONTRIBUIÇÕES PARA A PRÁTICA E RESULTADOS ALCANÇADOS}

A primeira CEE do HUCFF teve a sua legitimidade reconhecida mediante transparente processo eleitoral, podendo, assim, assumir a grande responsabilidade de representar o COREN/RJ dentro da instituição. Alguns desafios tiveram que ser superados logo na implementação, porém pôde-se contar com o apoio e orientações do COREN-RJ sobre legislação e nos processos éticos. Obteve-se, ainda, a colaboração da Divisão de Enfermagem do HUCFF para a estruturação da CEE, rompendo com as barreiras que poderiam dificultar o desempenho da comissão.

Podemos afirmar que, após a implementação da CEE, os profissionais de enfermagem tiveram mais oportunidades para conhecer os seus direitos e deveres dentro da prática profissional, tendo garantia de voz e defesa perante os conflitos vividos na instituição. Nesse sentido, a CEE, sempre de forma imparcial, prioriza o caráter conciliatório nas abordagens.

A capacitação da equipe eleva a capacidade de resolução de problemas com eficiência e fundamentação teórica, além de incentivar a discussão dos temas éticos e bioéticos no espaço hospitalar. Com isso, ampliam-se o conhecimento dos profissionais e a busca pela educação permanente e continuada de todo o corpo da enfermagem.

\section{CONSIDERAÇÕES FINAIS}

É importante dar voz aos trabalhadores da enfermagem para se construir uma prática ética imbuída de um olhar atento ao ambiente de trabalho, que procure tratar com respeito os problemas morais frequentemente vivenciados no cotidiano da enfermagem. A implantação da CEE foi o primeiro 
passo dado no esforço de alcançar a equidade de tratamento entres os profissionais, ouvindo, registrando e direcionando soluções com base na ética profissional elevada.

Observa-se que a realidade local não difere da realidade do país, permeada pela escassez de recursos humanos e insumos, limitação de acesso da população aos serviços, bem como conflitos de ordem interpessoal produzidos e agravados por tais carências. Acredita-se-assim, que este relato de experiência possa servir como modelo relevante para outras comissões. 


\section{REFERÊNCIAS}

CONSELHO FEDERAL DE ENFERMAGEM. Resolução no 311, de 6 de novembro 2007. Aprovar o novo Código de Ética dos Profissionais de Enfermagem. Brasília, DF: COFEN, 2007. Disponível em: http://www.cofen.gov.br/resoluo-cofen-3112007_4345.html. Acesso em: 10 abr. 2021.

CONSELHO FEDERAL DE ENFERMAGEM. Resolução $\mathbf{n}^{\circ}$ 564, de 6 de novembro de 2017. Aprovar o novo Código de Ética dos Profissionais de Enfermagem. Brasília, DF: COFEN, 2017. Disponível em: http://www.cofen.gov.br/resolucao-cofen-no-5642017_59145.html. Acesso em: 20 mar. 2021.

CONSELHO FEDERAL DE ENFERMAGEM. Resolução $\mathbf{n}^{\circ}$ 593, de 05 de novembro de 2018. Normatiza a criação e funcionamento das Comissões de Ética de Enfermagem-CEE nas Instituições com Serviço de Enfermagem. Brasília, DF: COFEN, 2018. Disponível em: http://www.cofen.gov.br/resolucao-cofen-no-593-2018_66530.html. Acesso em: 18 abr. 2018.

CONSELHO REGIONAL DE ENFERMAGEM DO RIO DE JANEIRO. Manual para implantação de comissão de ética de enfermagem nas instituições de saúde. Rio de Janeiro: COREN/RJ, 2015. Disponível em: http://rj.corens.portalcofen.gov.br/wp-content/uploads/2015/02/MANUAL-DEIMPLANTA\%C3\%83\%E2\%80\%A1\%C3\%83\%C6\%92O-CEE.pdf. Acesso em: 5 fev. 2021.

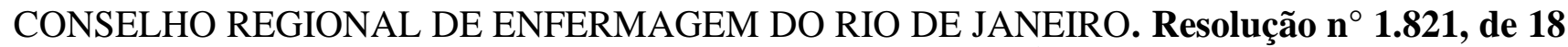
de outubro de 2012. Dispõe sobre a criação de Comissão de Ética de Enfermagem Institucional. Rio de Janeiro: COREN/RJ, 2012. Disponível em: https://docplayer.com.br/12977490-Decisao-corenrj-n-o-1821-2012.html. Acesso em: 7 mar. 2021. 